\title{
Changes of Standard Physiological-Perceptual Markers and Circulating MicroRNAs in Response to Tennis Match-Play: A Case Report of Two Elite Players
}

\author{
by \\ Matthias Wilhelm Hoppe ${ }^{1}$, Christian Baumgart ${ }^{1}$, Thomas Hilberg², \\ Jürgen Freiwald ${ }^{1}$, Udo Frank Wehmeier ${ }^{2}$
}

This study aimed to describe the acute changes of both standard physiological-perceptual markers and circulating microRNAs in response to tennis match-play in a detailed case report. Two elite male baseliners with comparable tennis experience were tested for anthropometric and fitness related variables and played $2 \mathrm{~h}$ of match-play on a red-clay court. The changes of standard physiological-perceptual markers including the heart rate, lactate concertation, creatine kinase activity, urea concentration and rating of perceived exertion as well as circulating microRNA-133a, -486 and -126 expression rates were examined at 10 different time-points (i.e., pre, during and up to $24 \mathrm{~h}$ post match-play). Player 2 had lower fitness related variables, but a higher heart rate, lactate concentration, creatine kinase activity and rating of perceived exertion during play than player 1. Player 2 showed an increase in all microRNAs $(\leq 3.83$-fold), most evident post match-play, whereas player 1 demonstrated a decrease $(\leq 0.41-$ fold $)$. The time-course in the changes of all standard physiological-perceptual markers was similar in both players, whereas this of the microRNAs was different. It was concluded that the relative changes of the circulating microRNA-133a, -486 and 126 expression rates of both players differed in response to tennis match-play with respect to the experienced physiological-perceptual stress and the underlying fitness level. Therefore, circulating microRNAs can serve as additional biomarkers for tennis exercise physiology and may be assessed together with standard markers to conclude whether key cellular regulatory processes were induced in response to match-play.

Key words: activity profile, gene expression, genetics, maximum oxygen uptake, training, testing.

\section{Introduction}

During the past two decades, tennis has evolved to a highly physiological demanding sport (Fernandez-Fernandez et al., 2009). Keeping pace with this development requires specific training drills, for which knowledge concerning the physiological demands of match-play is essential (Kovacs, 2007). So far, many studies have focused on the activity profile and changes of standard physiological-perceptual markers such as the heart rate $(\mathrm{HR})$, oxygen uptake $\left(\mathrm{VO}_{2}\right)$, lactate concentration, creatine kinase (CK) activity, urea concentration or rating of perceived exertion (RPE) in response to match-play (Gomes et al., 2014; Hornery et al., 2007; MendezVillanueva et al., 2007; Smekal et al., 2001). This plethora of research has led to an evidence based framework of tennis exercise physiology summarized in reviews (Fernandez-Fernandez et al., 2009; Kovacs, 2007).

Recently, a new level of regulation of cellular processes has been identified. MicroRNAs (miRs) are $\sim 22$ nucleotides long non-coding RNAs

\footnotetext{
1 - University of Wuppertal, Department of Movement and Training Sience, Wuppertal, Germany.

2 - University of Wuppertal, Department of Sports Medicine, Wuppertal, Germany.
} 
that regulate the gene expression on a posttranscriptional level by multiple miR:mRNA interactions, leading to translation inhibition or mRNA degradation (Denham et al., 2014). MiRs modulate the expression of up to $60 \%$ of proteincoding genes (Friedman et al., 2009), play key mechanisms in a wide range of diseases, ageing processes as well as exercise related adaptations (Zacharewicz et al., 2013) and are expressed in a tissue specific manner (Lagos-Quintana et al., 2002). Since it became evident that miRs can be released into the circulation after exercises (Gallo et al., 2012; Kosaka et al., 2010), they have been recently proposed as novel biomarkers of exercise physiology (Aoi et al., 2013; Banzet et al., 2013; Mooren et al., 2014; Uhlemann et al., 2014).

In recent studies, the changes of three frequently studied miRs, namely the skeletal and cardiac muscle specific miR-133a, skeletal muscle specific miR-486 (e.g., involved both in the regulation of cell development, proliferation and differentiation) and endothelial specific miR-126 (e.g., involved in the regulation of angiogenesis), were shown to exhibit exercise dependent expression changes (Aoi et al., 2013; Mooren et al., 2014; Uhlemann et al., 2014). Uhlemann et al. (2014) showed that cycling for $4 \mathrm{~h}$ at $70 \%$ of the anaerobic threshold and performing an incremental test induced the expression of the miR-126, whereas miR-133a remained constant. In contrast, performing eccentric resistance exercises with $3 \times 15$ repetitions led to an increase of the miR-133a with unchanged miR-126. Moreover, running a marathon enhanced both the miR-126 and miR-133a. Aoi et al. (2013) demonstrated that cycling for $60 \mathrm{~min}$ at $70 \%$ of $\mathrm{VO}_{2 \max }$ decreased the miR-486. Interestingly, the changes of the miR-486 were correlated with the level of $\mathrm{VO}_{2 \max }$. Mooren et al. (2014) revealed that running a marathon increased the miR-133a and CK activity. Noteworthy, the changes of the miR-133a were correlated with those of the CK activity and with the level of $\mathrm{VO}_{2 \max }$.

Overall, the findings from all mentioned previous studies provide unique inside into exercise physiology on a molecular level and support the use of circulating miRs as novel biomarkers. However, in most studies, no standard physiological-perceptual markers were co-analyzed and most research designs were based on pre/post measurements, and therefore, did not include the changes of miRs during the sportive load. Importantly, all previous studies have also indicated that there exist large betweensubject variations in the miR-responses, making it difficult to obtain meaningful main effects (Hecksteden et al., 2015). Consequently, and as a pragmatic alternative, this study aimed to describe the acute changes of both standard physiological-perceptual markers and circulating miRs in response to tennis match-play in a detailed case report. Here, we describe these changes in two elite players grounded on multiple time-points of measurement, also during matchplay, and thereby, providing first time-courses in the changes of both marker settings in response to exercise.

\section{Material and Methods}

\section{Participants}

Two elite male tennis players with comparable tennis experience were recruited to participate in this study (Table 1). Both athletes had been playing tennis for over 20 years, had been training on-court three and off-court two times per week during the last five years, had the highest German tennis ranking, were righthanded and preferred defensive play from the baseline. None of the players had any current or former chronic diseases, history of smoking or current use of medications. The players were informed of the purpose, procedures as wel as potential risks of the study and written informed consent was obtained. All procedures were approved by the Ethics Committee of the University of Wuppertal and were conducted in accordance with the Declaration of Helsinki.

\section{Experimental design}

Figure 1a shows the experimental design of the study. All testing procedures were conducted on two sessions during the first week after completing the outdoor-season. On the first session, the players were examined in the laboratory for body height, mass, fat and fat-free mass as well as vertical jump height, peak torque of the knee extensor muscles, overhead medicine ball throw distance, one repetition maximum bench press, time to exhaustion, $V_{2}$ max, ventilatory threshold and running economy. These variables were assessed to describe the anthropometric characteristics and fitness levels of the players (Fernandez-Fernandez et al., 2014). 
On the second session, the players performed $2 \mathrm{~h}$ of match-play. The match was filmed to evaluate the activity profile, reflecting, at least in part, the intensity and technical-tactical level of play (Mendez-Villanueva et al., 2007). Pre, during and up to $24 \mathrm{~h}$ post match-play, standard physiological-perceptual markers including the HR, lactate concentration, CK activity, urea concentration and RPE as well as the circulating miR-133a, -486 and -126 expression rates were measured. Therefore, capillary blood samples from the earlobe were collected at 10 different time-points (Figure 1b). During the match, blood samples were taken throughout selected changeover breaks. The players were asked to report to laboratory testing well-rested, to have refrained from exercise for the prior two days and to perform no exercises the four days between laboratory testing and the tennis match. Before and after the match, the players received the same carbohydrate-rich breakfast and lunch, respectively (Figure $1 b$ ). Since both players were habituated to caffeine, they were allowed to drink coffee for their breakfast.

\section{Laboratory testing procedures}

Body fat and fat-free mass

Body fat and fat-free mass of the players were examined using a 4-point bioelectrical impedance analysis in a supine position (Bodystat, QuadScan 4000, Douglas, United Kingdom). Thereafter, the players warmed-up for $10 \mathrm{~min}$ including self-paced running on a treadmill. The players were given at least $15 \mathrm{~min}$ recovery between each of the following testing procedures.

Vertical jump height

The players performed three maximal counter movement jumps during which the arms were free to move. The jump height was determined using a force plate (Kistler, 9287BA, Winterthur, Switzerland). Each jump was followed by a 2 min recovery period and the highest jump was recorded for further analysis.

Peak torque of the knee extensor muscles

The players performed three maximal isometric knee extensions over $6 \mathrm{~s}$. The exercises were performed with the dominant leg at a knee flexion angle of $60^{\circ}$. The measurement was conducted in a seated position using an isokinetic dynamometer (Cybex Humac, NORM, California, USA). Each exercise was separated by a 3 min rest period and the highest torque was recorded.

Overhead medicine ball throw distance

The players executed three maximal overhead throws with a $2 \mathrm{~kg}$ medicine ball. The players stood in front of a line, moved the ball back behind the head and then, threw the ball forward without crossing the line. Each throw was followed by a $2 \mathrm{~min}$ rest period and the farthest distance was recorded for further analysis.

One repetition maximum bench press

The players executed up to four submaximal to maximal bench press exercises using a competition style bar (Gym80, International Sygnum Basic, Gelsenkirchen, Germany). After warming up with individual weights, the load was increased in $5 \mathrm{~kg}$ increments until a one repetition maximum was reached. After each attempt, the players had a 3 min recovery period and the maximum load was recorded.

Time to exhaustion, maximum oxygen uptake, ventilatory threshold and running economy

The players performed an incremental running test on a motorized treadmill $(\mathrm{H} / \mathrm{P}$ Cosmos, Pulsar, Nussdorf-Traunstein, Germany). Briefly, the initial two increments consisted of running for $4 \mathrm{~min}$ at $10 \mathrm{~km} \cdot \mathrm{h}^{-1}$ at an inclination of $1 \%$ and $5 \%$, respectively. Thereafter, the running speed was increased every $2 \mathrm{~min}$ by $1 \mathrm{~km} \cdot \mathrm{h}^{-1}$ until exhaustion was reached. The respiratory gas exchange and HR were measured using an open circuit breath-by-breath gas analyzing system (Ganshorn, PowerCube-Ergo, Niederlauer, Germany) and a chest belt (Suunto, t6, Vantaa, Finland), respectively. $\mathrm{VO}_{2 \max }$ was considered to be reached, if three of the following four criteria were fulfilled: a plateau in $\mathrm{VO}_{2}$ (increase $<2$ $\mathrm{ml} \cdot \mathrm{min}^{-1} \cdot \mathrm{kg}^{-1}$ ) despite an increase in the workload, a respiratory exchange ratio $>1.15$, a minimum of $95 \%$ of an age-predicted maximal HR (220-age) or a capillary blood lactate concentration $>8.0$ mmol. $\mathrm{l}^{-1}$. The ventilatory threshold was determined by two investigators using the modified V-slope method. Running economy was assessed using the average $\mathrm{VO}_{2}$ of the last $60 \mathrm{~s}$ at $10 \mathrm{~km} \cdot \mathrm{h}^{-1}$ with $1 \%$ inclination and was expressed as oxygen cost per distance covered.

\section{Tennis match-play}

The players were asked to play $2 \mathrm{~h}$ of match-play at their best level. The match was 
conducted on a red-clay court according to the rules of the ITF. Before the match, the players warmed-up for $10 \mathrm{~min}$ with ground strokes, volleys, overhead strokes and serves. Three new balls (Dunlop, Fort Tournament, Blackpool, United Kingdom) were used for the match. During play, the players retrieved their own balls, counted the games won and were allowed to drink water ad libitum. The match was performed at 10:00 a.m. and the weather conditions during play were ambient $\left(18-21^{\circ} \mathrm{C}\right.$ and $36-44 \%$ humidity).

Activity profile

The tennis match was filmed by two video cameras (Sony, DCR-SR190, Tokyo, Japan). The following variables were determined from the video: duration of the rallies, the number of strokes per rally, rest time between the rallies, a work:rest ratio and effective playing time (Mendez-Villanueva et al., 2007).

Changes of the standard physiological-perceptual markers

The HRs were recorded using short-range telemetry (Suunto, t6, Vantaa, Finland) and analyzed in relation to the maximal values achieved during the incremental test. The lactate concentrations were determined from $20 \mu \mathrm{l}$ capillary blood samples, which were collected with heparinized capillaries and analyzed using an electro-enzymatic analyzer (EKF-diagnostics, Biosen C_line Sport, London, United Kingdom). CK activities and urea concentrations were determined from $32 \mu \mathrm{l}$ capillary blood samples, which were collected with capillaries connected to EDTA-powdered tubes (Sarstedt, Microvette CB 300, Nümbrecht, Germany) and analyzed using a spectrophotometer (Roche Diagnostics, Reflotron, Mannheim, Germany). From each blood sample, the lactate, CK and urea analyses were performed in duplicate and the mean was recorded. The RPEs were determined using the 15-category scale of Borg.

Changes of the circulating microRNAs

The changes of the circulating miR-133a, 486 and -126 expression rates were analyzed according to the methodology developed by Wehmeier and Hilberg (2014). Similarly to lactate analyses, the changes of the circulating miRs were determined from $20 \mu \mathrm{l}$ capillary blood samples, which were collected with EDTA-coated capillaries (Sarstedt) and added into tubes filled with $1 \mathrm{ml}$ PeqGold reagents (PeqLab, Erlangen, Germany). Immediately after collecting, the tubes were cooled at $-20^{\circ} \mathrm{C}$ and frozen on the next day at $80^{\circ} \mathrm{C}$ for later analyses. All following procedures were performed according to the instructions of the manufactures. The total RNA extraction was performed using a PeqGold Extration Kit (PeqLab). The extracted RNA samples were then analyzed using a StepOne real time PCR unit (Life Technologies, Darmstadt, Germany) applying TaqMan Detection Kits (Life Technologies). The $18 \mathrm{~S}$ rRNA was used as an internal standard and was determined with an Eukaryotic 18S rRNA Endogenous Control Kit (Life Technologies, $\mathrm{FAM}^{\mathrm{TM}} / \mathrm{MGB}$ probe, non-primer limited). Each sample was quantified in duplicate and the mean was recorded. Based on the $\mathrm{C}_{\mathrm{T}}$ values, the copy numbers of the circulating miRs and the 18S rRNA were calculated. The ratios were normalized to the pre match-play values and the relative changes ( $x$-fold versus pre) of the circulating miRs were calculated.

\section{Results}

Anthropometric characteristics and fitness levels

Table 1 shows the variables describing the anthropometric characteristics and fitness levels of both players. Player 2 had lower anthropometric and fitness related variables than player 1.

\section{Activity profile of the tennis match}

The mean \pm SD (Range) values for the duration of the rallies, number of strokes per rally and rest time between the rallies were $14.1 \pm 11.7 \mathrm{~s}$ (1.1-72.3), $7.2 \pm 5.6(1-40)$ and $25.6 \pm 7.9$ s (11.639.5), respectively. The values for the work:rest ratio and effective playing time were $1: 2$ and $27.2 \%$, respectively. Player 1 won in total one game more than player 2 (i.e., 11:10).

\section{Changes of the standard physiological-perceptual markers}

Table 2 summarizes the HRs, lactate concentrations, CK activities, urea concentrations and RPEs of both players in response to matchplay. With exception of lower urea concentrations, player 2 had higher HRs, lactate concentrations, CK activities and RPEs during play and also at the majority of the other time-points than player 1 . Figure 2 demonstrates the relative changes of the HRs, lactate concentrations, CK activities and urea concentrations of both players. Player 2 also had 
higher relative changes in these markers compared to player 1 . However, the time-course in the relative changes of the HRs, lactate concentrations, $\mathrm{CK}$ activities and urea concentrations was similar in both players.

(a)
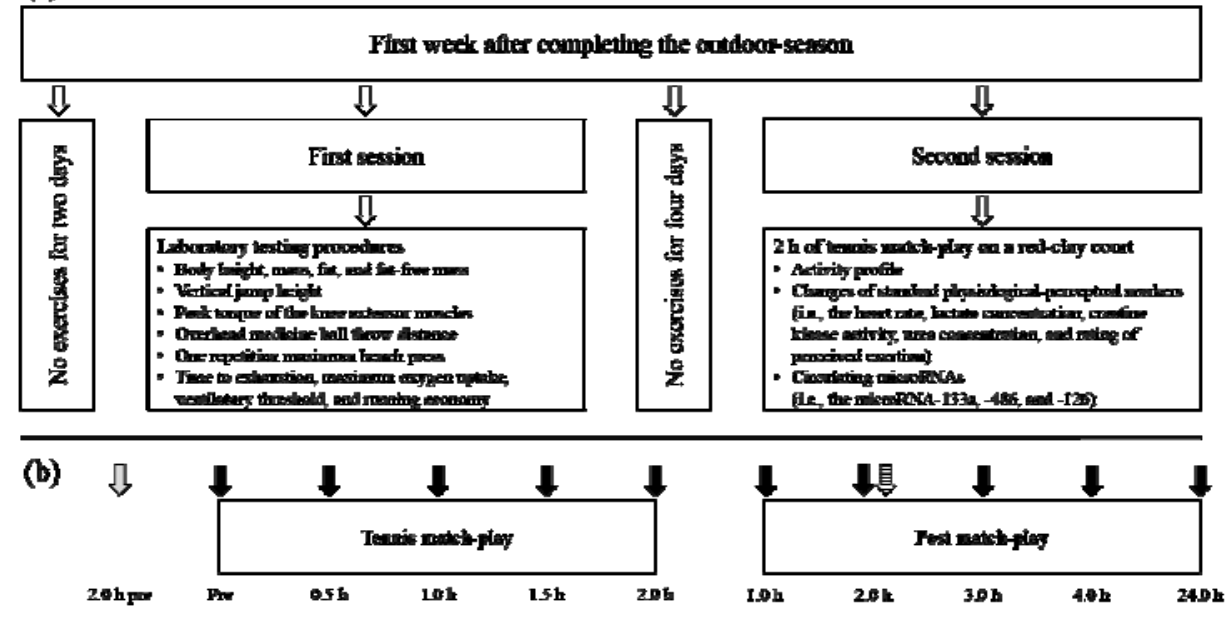

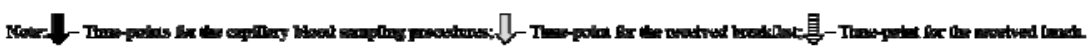

Figure 1

Research design of the study (a) as well as time-points of capillary

blood sampling procedures and received meals $(b)$

Table 1

Tennis experience, anthropometric characteristics and fitness levels of both elite tennis players

\begin{tabular}{|c|c|c|c|}
\hline Variable & Player 1 & Player 2 & Diff. (\%) \\
\hline Tennis experience (years) & 22 & 21 & -4.5 \\
\hline Training sessions per week & 5 & 5 & 0.0 \\
\hline German ranking (1-23) & 1 & 1 & 0.0 \\
\hline Preferred playing strategy & Defensive & Defensive & $\mathrm{n} / \mathrm{a}$ \\
\hline Age (years) & 29 & 26 & -10.3 \\
\hline Body height (cm) & 189 & 178 & -5.8 \\
\hline Body mass (kg) & 92.7 & 69.8 & -24.7 \\
\hline Body fat (\%) & 12.2 & 13.5 & +10.7 \\
\hline Body fat-free mass $(\mathrm{kg})$ & 81.4 & 60.4 & -25.8 \\
\hline Peak torque of the knee extensor muscles $(\mathrm{N} \cdot \mathrm{m})$ & 350 & 250 & -28.6 \\
\hline Vertical jump height $(\mathrm{cm})$ & 48.5 & 29.0 & -40.2 \\
\hline One repetition maximum bench press $(\mathrm{kg})$ & 95 & 75 & -21.1 \\
\hline Overhead medicine ball throw distance $(\mathrm{m})$ & 14.4 & 10.8 & -25.0 \\
\hline Time to exhaustion (s) & 905 & 780 & -13.8 \\
\hline Maximum oxygen uptake $\left(1 \cdot \mathrm{min}^{-1}\right)$ & 4.8 & 3.3 & -31.2 \\
\hline Maximum oxygen uptake $\left(\mathrm{ml} \cdot \mathrm{kg}^{-1} \cdot \mathrm{min}^{-1}\right)$ & 51.8 & 47.3 & -8.7 \\
\hline Ventilatory threshold $\left(\% \mathrm{VO}_{2 \max }\right)$ & 87.5 & 78.0 & -10.9 \\
\hline Running economy $\left(\mathrm{ml} \cdot \mathrm{kg}^{-1} \cdot \mathrm{km}^{-1}\right)$ & 218 & 207 & -5.0 \\
\hline
\end{tabular}

Note: Diff. $(\%)=$ Differences are calculated from the perspective of player $2 ; n / a=$ not available. 
Table 2

Heart rates, lactate concentrations, creatine kinase activities, urea concentrations and ratings of perceived exertion of both elite players in response to tennis match-play

\begin{tabular}{|c|c|c|c|c|c|c|c|c|c|c|}
\hline \multirow[t]{3}{*}{ Time-point } & \multicolumn{2}{|c|}{$\begin{array}{c}\text { Hear rate } \\
(\%)\end{array}$} & \multicolumn{2}{|c|}{$\begin{array}{c}\text { Lactate } \\
\text { concentration } \\
\left(\mathrm{mmol} \cdot \mathrm{l}^{-1}\right)\end{array}$} & \multicolumn{2}{|c|}{$\begin{array}{c}\text { Creatine } \\
\text { kinase activity } \\
\left(\mathrm{U} \cdot \mathbf{l}^{-1}\right)\end{array}$} & \multicolumn{2}{|c|}{$\begin{array}{c}\text { Urea } \\
\text { concentration } \\
\left(\mathrm{mg} \cdot \mathrm{dl} \mathbf{l}^{-1}\right)\end{array}$} & \multicolumn{2}{|c|}{$\begin{array}{c}\text { Rating of } \\
\text { perceived } \\
\text { exertion }(6-20)\end{array}$} \\
\hline & Player & Player & Player & Player & Player & Player & Player & Player & Player & Player \\
\hline & 1 & 2 & 1 & 2 & 1 & 2 & 1 & 2 & 1 & 2 \\
\hline Pre & 31.7 & 40.6 & 1.4 & 0.9 & 341 & 551 & 53 & 31 & 7 & 6 \\
\hline $0.5 \mathrm{~h}$ & 83.0 & 85.7 & 2.5 & 4.7 & 322 & 554 & 55 & 32 & 16 & 16 \\
\hline $1.0 \mathrm{~h}$ & 85.5 & 85.5 & 1.9 & 3.1 & 304 & 523 & 54 & 31 & 16 & 17 \\
\hline $1.5 \mathrm{~h}$ & 83.2 & 83.4 & 2.7 & 3.5 & 207 & 457 & 58 & 34 & 15 & 17 \\
\hline $2.0 \mathrm{~h}$ & 86.8 & 84.6 & 2.5 & 2.6 & 223 & 438 & 61 & 39 & 16 & 18 \\
\hline $1.0 \mathrm{~h}$ post & $\mathrm{n} / \mathrm{a}$ & $\mathrm{n} / \mathrm{a}$ & 0.8 & 0.9 & 164 & 195 & 57 & 33 & 9 & 10 \\
\hline $2.0 \mathrm{~h}$ post & $\mathrm{n} / \mathrm{a}$ & $\mathrm{n} / \mathrm{a}$ & 1.1 & 0.6 & 443 & 795 & 55 & 31 & 8 & 8 \\
\hline $3.0 \mathrm{~h}$ post & $\mathrm{n} / \mathrm{a}$ & $\mathrm{n} / \mathrm{a}$ & 0.9 & 0.6 & 522 & 231 & 53 & 31 & 7 & 7 \\
\hline $4.0 \mathrm{~h}$ post & $\mathrm{n} / \mathrm{a}$ & $\mathrm{n} / \mathrm{a}$ & 1.0 & 0.7 & 227 & 127 & 54 & 32 & 6 & 7 \\
\hline $24.0 \mathrm{~h}$ post & $\mathrm{n} / \mathrm{a}$ & $\mathrm{n} / \mathrm{a}$ & 1.2 & 0.8 & 264 & 358 & 55 & 34 & 6 & 6 \\
\hline
\end{tabular}

Note: Heart rates during match-play are presented as the mean of the associated period; n/a = not available.
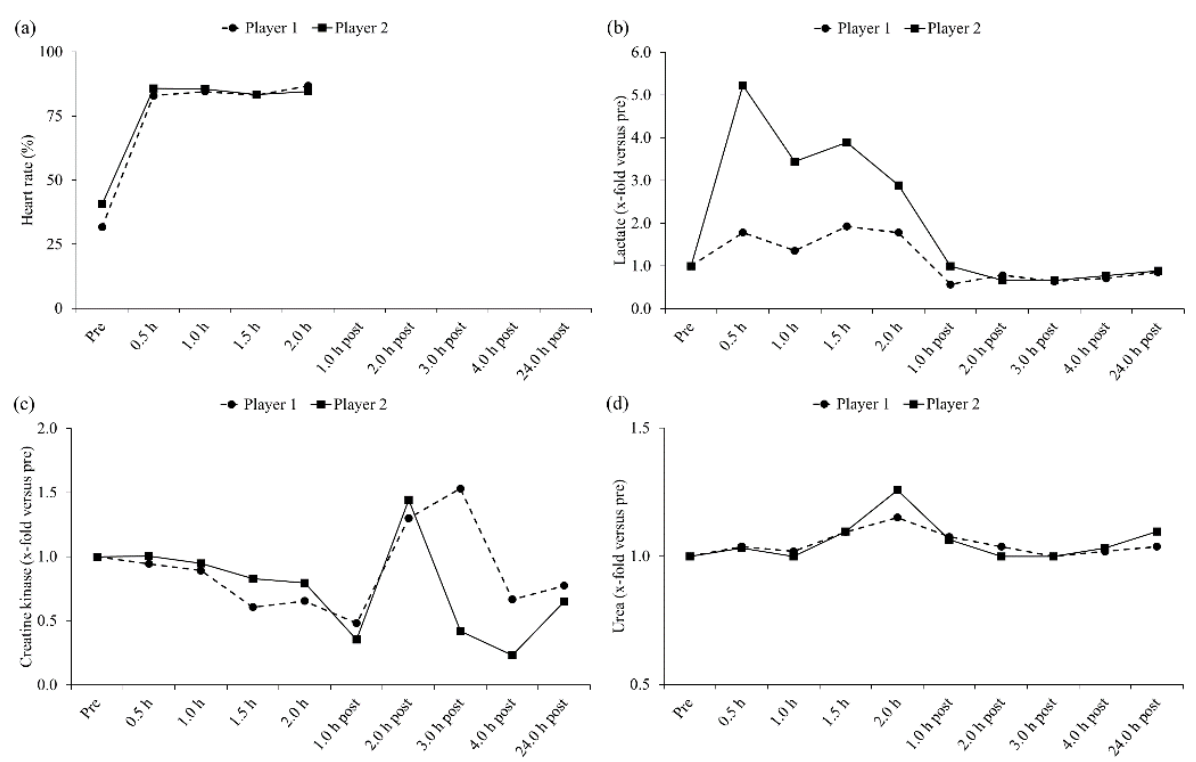

Figure 2

Relative changes of heart rates (a), lactate concentrations (b), creatine kinase activities

(c) and urea concentrations (d) of both elite players in response to tennis match-play 


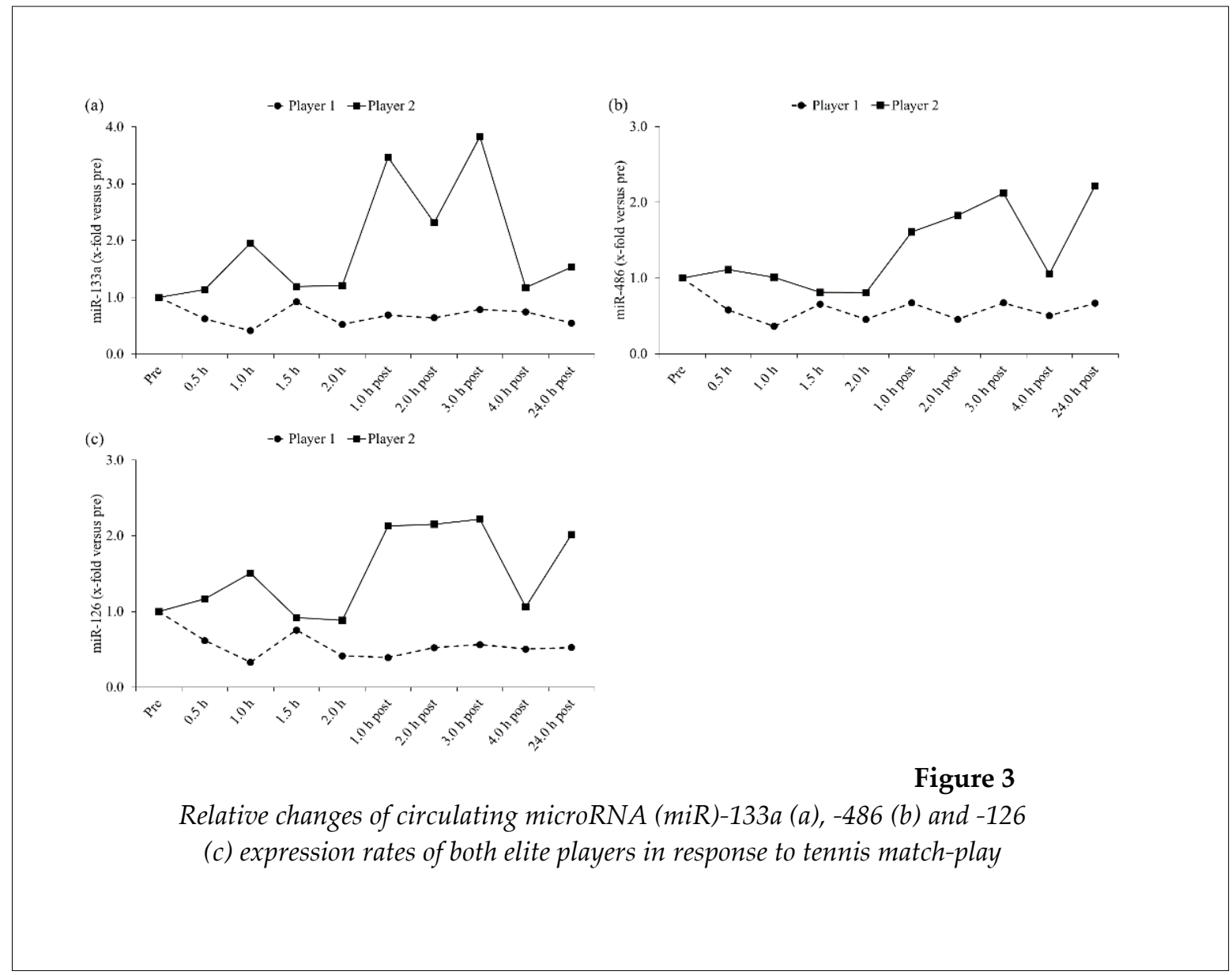

\section{Changes of the circulating microRNAs}

The mean $C_{T}$ values of the miR-133a, -486 and -126 were for both players 33.7 and $32.5,18.2$ and 17.9 and 20.6 and 20.2, respectively. Figure 3 shows the relative changes of the circulating miR133a, -486 and -126 expression rates of both players in response to match-play. While player 2 showed an increase in the miR-133a ( $\leq 3.83$-fold), 486 ( $\leq 2.22$-fold) and -126 ( $\leq 2.22$-fold), most evident post match-play, player 1 demonstrated a decrease in these miRs ( $\leq 0.41$-fold, $\leq 0.36$-fold and $\leq 0.33$-fold, respectively). In contrast to the standard physiological-perceptual markers, the time-course in the relative changes of the miR133a, -486 and -126 was different in both players.

\section{Discussion}

Our major findings were as follows: 1.) the relative changes of the circulating miR-133a, 486 and -126 expression rates of both players differed in response to tennis match-play with respect to the experienced physiological- perceptual stress and the underlying fitness level; and 2.) the time-course in the changes of all standard physiological-perceptual markers was similar in both players, whereas this of the miRs was different.

In this study, the mean duration of the rallies (14.1 s), the number of strokes per rally (7.2) and rest time between the rallies (25.6 s) were higher than the ranges reported in one review (5$10 \mathrm{~s}, 2.5-3.0$ and $10-20 \mathrm{~s}$, respectively) (FernandezFernandez et al., 2009). The main explanations for these discrepancies may be that both players preferred defensive play from the baseline, made only few unforced errors and that we did not investigate a real competition (Smekal et al., 2001). However, the work:rest ratio (1:2) and effective playing time $(27.2 \%)$ were in line with the ranges stated before (1:1-1:4 and 20.0-30.0\%, respectively) (Fernandez-Fernandez et al., 2009). The HRs (83.0-86.8 and 83.4-85.7\%), lactate concentrations (1.9-2.7 and 2.6-4.7 $\left.\mathrm{mmol} \cdot \mathrm{l}^{-1}\right)$ and RPEs (15-16 and 16-18) of both players examined 
during match-play (Table 2) were also higher than the ranges reported earlier $(70.0-80.0 \%, 1.8-2.8$ mmol. $\mathrm{l}^{-1}$ and 11-14, respectively) (Fernandez-Fernandez et al., 2009). These observations may be related to the specific characteristics of the present activity profile (e.g., long rally duration), indicating that the physiological-perceptual stress might be correlated with the characteristics of the activityprofile (Mendez-Villanueva et al., 2007). While the peak CK activity of player 1 assessed post matchplay (522 U.1-1) was only slightly higher than the values reported before (414-498 U.1-1) (Gomes et al., 2014; Hornery et al., 2007), the peak value of player 2 was clearly higher (795 U.1-1). Furthermore, the peak urea concentration of player $1\left(61 \mathrm{mg}^{\left.-1 l^{-1}\right)}\right.$ was higher, whereas the peak value of player $2\left(39 \mathrm{mg} \cdot \mathrm{dl}^{-1}\right)$ was in line with the

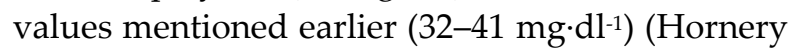
et al., 2007). Since both players won a comparable number of games (i.e., 11:10), it can be summarized that the tennis match was wellbalanced, characterized by an activity-profile typical for two elite baseliners and played intensively enough to noticeably induce physiological-perceptual stress.

This is the first study to investigate relative changes of circulating miR-133a, -486 and -126 expression rates of two elite tennis players in response to match-play. For these analyses, we applied a recent validated and minimally-invasive methodology, requiring only $20 \mu \mathrm{l}$ capillary blood samples (Wehmeier and Hilberg, 2014). The mean $\mathrm{C}_{\mathrm{T}}$ values of the players' miR-133a (33.7 and 32.5) and miR-486 (18.2 and 17.9) differed from those reported in a previous study (35.4 for miR-133a and 25.1 for miR-486) (Aoi et al., 2013). This means that the overall expression rates of the examined miRs were higher in our study. No data to compare were available for the $\mathrm{C}_{\mathrm{T}}$ values of the miR-126. The observed differences in the $C_{T}$ values may be related to the different blood samples used (Wehmeier and Hilberg, 2014). While we analyzed capillary whole blood, venous blood serum had been examined in a previous study (Aoi et al., 2013). In contrast to blood serum, capillary blood still contains cells, which may contribute to the differences in observed $C_{T}$ values (Wehmeier and Hilberg, 2014). Since all $C_{\mathrm{T}}$ values of this study were below the cut-off value of 35 PCR cycles, where circulating miRs are difficult to quantify (Aoi et al., 2013), our results are appropriate and allow further discussions.

Our first major finding was that the relative changes of the circulating miR-133a, -486 and -126 expression rates of both players clearly differed in response to match-play (Figure 3). While player 2 showed an increase in all miRs ( $\leq 3.83$-fold), most evident post match-play, player 1 demonstrated a decrease ( $\leq 0.41$-fold). One explanation for the increases in the miRs of player 2 may be that this player had lower fitness related variables than player 1 (Table 1). This lower fitness level may account for the higher HRs, lactate concentrations and RPEs assessed during match-play as well as higher peak CK activity assessed post match-play (Table 2 and Figure 2). Therefore, it is reasonable to assume that player 2 experienced higher physiological-perceptual stress in response to match-play (FernandezFernandez et al., 2009; Kovacs, 2007). The higher physiological-perceptual stress may have led to the activation of intracellular signal transduction pathways, causing the up-regulation of de novo miR transcription (Baggish et al., 2011; Mooren et al., 2014) or processing of pre-existing, but inactive pre-mature miRs and their transport into the circulation (Baggish et al., 2011; Mooren et al., 2014). A further consideration could be that physiological-perceptual stress led to the mechanical damage of the skeletal and cardiac muscle or endothelial cell membranes. From these damaged cells, the miR-133a, -486 and -126 could have been released into the circulation (Banzet et al., 2013; Mooren et al., 2014; Uhlemann et al., 2014). An explanation for the decreases in the miRs of player 1 may be that this player had higher urea concentrations compared to player 2 (Table 2). Since elevated urea concentrations are associated with an enhanced protein catabolism (Haralambie and Berg, 1976), it could be expected that the decreases in the miRs may be caused by the destruction of the protein-complexes of the exosome vehicles, leading to the degradation of the miRs by RNases (Aoi et al., 2013). Two additional explanations may be that the circulating miRs were taken up by other cells, as a result of cell-cell signaling processes (Kosaka et al., 2013; Turchinovich et al., 2013) or that they were less released from the tissues (Aoi et al., 2013; Aoi and Sakuma, 2014). Overall, our findings show that the changes of the circulating 
miR-133a, -486 and -126 expression rates of both players differed in response to match-play with respect to the experienced physiologicalperceptual stress and the underlying fitness level. These assumptions are supported by the findings of few previous studies (Aoi et al., 2013; Mooren et al., 2014; Uhlemann et al., 2014) and facilitate, in combination with our applied minimally-invasive methodology (Wehmeier and Hilberg, 2014), the application of the examined miRs as promising biomarkers of tennis exercise physiology.

Our second major finding was that the time-course in the changes of all standard physiological-perceptual markers was similar in both players (Table 2 and Figure 2), whereas this of the circulating miRs was different (Figure 3). An explanation may be that our tennis match was well-balanced, which may have led to similar patterns in the changes of the experienced physiological-perceptual stress of both players. However, grounded on the underlying fitness level, the parallelism of the physiologicalperceptual stress might have led to different cellular regulatory processes, as seen by the diverse patterns in the changes of the miRs. These assumptions show that our applied setting of standard markers will be useful to evaluate the physiological-perceptual stress in response to match-play; however, no information whether key cellular regulatory processes were induced had been provided (Baggish et al., 2014). On the other hand, the patterns in the changes of the miRs may indicate that more cell regulatory mechanisms were induced in player 2 than in player 1 (Figure 3). Since it was shown that the changes of miRs may allow to distinguish between young men with high and low capacities to adapt to resistance exercises (Davidsen et al., 2011), it could be hypothesized that we identified player 2 as a "responder" and player 1 as a "nonresponder" on the molecular level to the most specific tennis training - i.e., match-play. To summarize, our settings of the standard markers and the circulating miRs may be assessed together to monitor the physiological-perceptual stress and to conclude whether key cellular regulatory processes are induced in response to match-play. Only the combination of both marker settings may allow to fine-tune key adaptation processes in response to training regimes.

Besides these major findings, we observed two unexpected outcomes. Unique to the literature, we examined $\mathrm{CK}$ activities and urea concentrations not only pre and post tennis match-play (Gomes et al., 2014; Hornery et al., 2007) or according to conditioning procedures (Ziemann et al., 2012; Ziemann et al., 2013) and sport-medical investigations (Keul et al., 1991), but also during the sportive load. Since it had been reported that CK activities increased after match-play (Gomes et al., 2014; Hornery et al., 2007), as in our study, it was astonishing that CK activities clearly decreased during match-play in both players (Table 2 and Figure 2c). While the mechanisms are beyond the scope of this study, they should be discussed briefly. A decrease in CK activities during match-play might be mediated by the pinocytosis of the enzyme via macrophages. Then, the enzyme is hydrolyzed into amino acids, which are released back into the circulation (Alberts et al., 2008). In exercising animals, it has also been shown that the enzyme is transported backwards into the lymphatic system, where it is potentially inactivated by free calcium (Volfinger et al., 1994). Therefore, the observed decrease in CK activities could have been caused by different exercise induced metabolic or transport-driven clearance mechanisms throughout different compartments. Furthermore, our results show that urea concentrations increased after $1 \mathrm{~h}$ of match-play in both players (Table 2 and Figure 2d). This finding is in line with the results of a previous study, showing that urea concentrations increased after $60-70 \mathrm{~min}$ of different endurance related exercises in trained athletes (Haralambie and Berg, 1976). These findings suggest that there could be a need for an enhanced protein catabolism and stimulated gluconeogenesis due to elevated energy requirements after $1 \mathrm{~h}$ of match-play.

While this study revealed new insights into the changes of standard physiologicalperceptual markers and circulating miRs in response to tennis match-play, our findings were limited by the applied case report design that allows no generalization. Instead, the results of the present study provide a helpful framework for future studies in this widely unknown research field. More studies to investigate the release and clearance mechanisms of circulating miRs in response to different standardized protocols (e.g., endurance versus resistance exercises at 
individualized loads) examining athletes from diverse backgrounds (e.g., sex, age, training levels) are strongly warranted.

\section{Conclusions}

The relative changes of the circulating miR-133a, -486 and -126 expression rates of two elite tennis players differed in response to matchplay with respect to the experienced physiological-perceptual stress and the underlying fitness level. Therefore, the examined miRs can serve as promising biomarkers of tennis exercise physiology. Furthermore, the time-course

\section{Acknowledgements}

The authors are grateful to both players for their participation, Jutta Bornefeld and Katrin Schröder for their assistance during the data assessment as well as Joana Brochhagen for her linguistic comments.

\section{References}

Alberts B, Johnson A, Lewis J, Raff M, Keith R, Walter P. Molecular Biology of the Cell. New York: Garland Science 2008

Aoi W, Ichikawa H, Mune K, Tanimura Y, Mizushima K, Naito Y, Yoshikawa T. Muscle-enriched microRNA miR-486 decreases in circulation in response to exercise in young men. Front Physiol, 2013; 4: 1-7

Aoi W, Sakuma K. Does regulation of skeletal muscle function involve circulating microRNAs? Front Physiol, 2014; 5: 1-8

Baggish AL, Hale A, Weiner RB, Lewis GD, Systrom D, Wang F, Wang TJ, Chan SY. Dynamic regulation of circulating microRNA during acute exhaustive exercise and sustained aerobic exercise training. $J$ Physiol, 2011; 589: 3983-3994

Baggish AL, Park J, Min PK, Isaacs S, Parker BA, Thompson PD, Troyanos C, D'Hemecourt P, Dyer S, Thiel M, Hale A, Chan SY. Rapid upregulation and clearance of distinct circulating microRNAs after prolonged aerobic exercise. J Appl Physiol (1985), 2014; 116: 522-531

Banzet S, Chennaoui M, Girard O, Racinais S, Drogou C, Chalabi H, Koulmann N. Changes in circulating microRNAs levels with exercise modality. J Appl Physiol (1985), 2013; 115: 1237-1244

Davidsen PK, Gallagher IJ, Hartman JW, Tarnopolsky MA, Dela F, Helge JW, Timmons JA, Phillips SM. High responders to resistance exercise training demonstrate differential regulation of skeletal muscle microRNA expression. J Appl Physiol (1985), 2011; 110: 309-317

Denham J, Marques FZ, O'Brien BJ, Charchar FJ. Exercise: putting action into our epigenome. Sports Med, 2014; 44: 189-209

Fernandez-Fernandez J, Sanz-Rivas D, Mendez-Villanueva A. A review of the activity profile and physiological demands of tennis match play. Strength and Conditioning Journal, 2009; 31: 15-26

Fernandez-Fernandez J, Ulbricht A, Ferrauti A. Fitness testing of tennis players: how valuable is it? $\mathrm{Br} \mathrm{J}$ Sports Med, 2014; 48: 22-31

Friedman RC, Farh KK, Burge CB, Bartel DP. Most mammalian mRNAs are conserved targets of microRNAs. Genome Res, 2009; 19: 92-105

Gallo A, Tandon M, Alevizos I, Illei GG. The majority of microRNAs detectable in serum and saliva is concentrated in exosomes. PLoS One, 2012; 7: 1-5

Gomes RV, Santos RC, Nosaka K, Moreira A, Miyabara EH, Aoki MS. Muscle damage after a tennis match in young players. Biol Sport, 2014; 31: 27-32

Haralambie G, Berg A. Serum urea and amino nitrogen changes with exercise duration. Eur J Appl Physiol Occup Physiol, 1976; 36: 39-48 
Hecksteden A, Kraushaar J, Scharhag-Rosenberger F, Theisen D, Senn S, Meyer T. Individual response to exercise training - a statistical perspective. J Appl Physiol (1985), 2015; 118: 1450-1459

Hornery DJ, Farrow D, Mujika I, Young W. An integrated physiological and performance profile of professional tennis. Br J Sports Med, 2007; 41: 531-536

Keul J, Stockhausen W, Pokan R, Huonker M, Berg A. [Metabolic and cardiovascular adaptation and the performance of professional tennis players]. Dtsch Med Wochenschr, 1991; 116: 761-767

Kosaka N, Iguchi H, Yoshioka Y, Takeshita F, Matsuki Y, Ochiya T. Secretory mechanisms and intercellular transfer of microRNAs in living cells. J Biol Chem, 2010; 285: 17442-17452

Kosaka N, Yoshioka Y, Hagiwara K, Tominaga N, Katsuda T, Ochiya T. Trash or Treasure: extracellular microRNAs and cell-to-cell communication. Front Genet, 2013; 4: 1-8

Kovacs MS. Tennis physiology: training the competitive athlete. Sports Med, 2007; 37: 189-198

Lagos-Quintana M, Rauhut R, Yalcin A, Meyer J, Lendeckel W, Tuschl T. Identification of tissue-specific microRNAs from mouse. Curr Biol, 2002; 12: 735-739

Mendez-Villanueva A, Fernandez-Fernandez J, Bishop D, Fernandez-Garcia B, Terrados N. Activity patterns, blood lactate concentrations and ratings of perceived exertion during a professional singles tennis tournament. Br J Sports Med, 2007; 41: 296-300

Mooren FC, Viereck J, Kruger K, Thum T. Circulating microRNAs as potential biomarkers of aerobic exercise capacity. Am J Physiol Heart Circ Physiol, 2014; 306: 557-563

Smekal G, von Duvillard SP, Rihacek C, Pokan R, Hofmann P, Baron R, Tschan H, Bachl N. A physiological profile of tennis match play. Med Sci Sports Exerc, 2001; 33: 999-1005

Turchinovich A, Samatov TR, Tonevitsky AG, Burwinkel B. Circulating miRNAs: cell-cell communication function? Front Genet, 2013; 4: 1-10

Uhlemann M, Mobius-Winkler S, Fikenzer S, Adam J, Redlich M, Mohlenkamp S, Hilberg T, Schuler GC, Adams V. Circulating microRNA-126 increases after different forms of endurance exercise in healthy adults. Eur J Prev Cardiol, 2014; 21: 484-491

Volfinger L, Lassourd V, Michaux JM, Braun JP, Toutain PL. Kinetic evaluation of muscle damage during exercise by calculation of amount of creatine kinase released. Am J Physiol, 1994; 266: 434-441

Wehmeier UF, Hilberg T. Capillary earlobe blood may be used for RNA isolation, gene expression assays and microRNA quantification. Mol Med Rep, 2014; 9: 211-216

Zacharewicz E, Lamon S, Russell AP. MicroRNAs in skeletal muscle and their regulation with exercise, ageing, and disease. Front Physiol, 2013; 4: 1-11

Ziemann E, Olek RA, Kujach S, Grzywacz T, Antosiewicz J, Garsztka T, Laskowski R. Five-day whole-body cryostimulation, blood inflammatory markers, and performance in high-ranking professional tennis players. J Athl Train, 2012; 47: 664-672

Ziemann E, Zembron-Lacny A, Kasperska A, Antosiewicz J, Grzywacz T, Garsztka T, Laskowski R. Exercise training-induced changes in inflammatory mediators and heat shock proteins in young tennis players. J Sports Sci Med, 2013; 12: 282-289

\section{Corresponding author:}

\section{Matthias W. Hoppe}

University of Wuppertal, Department of Movement and Training Science

Fuhlrottstaße 10, 42119 Wuppertal, Germany

Phone: $+49(0) 2024393226$

E-mail: m.hoppe@uni-wuppertal.de 